\title{
Etiological Evaluation of Acute Confusional State in Elderly ( $\geq 60$ Years) Patients
}

\author{
Md. Motlabur Rahman ${ }^{1}$, Rakesh Panday ${ }^{2}$, Kamal Uddin Sohel ${ }^{3}$, Zuhayer Ahmed ${ }^{4}$, Shahana Khanam ${ }^{5}$, M A \\ Sattar Sarker ${ }^{6}$, Pratyay Hasan ${ }^{7}$, Md. Khairul Islam ${ }^{8}$
}

\begin{abstract}
:
Background: Acute confusional state or delirium is a frequent cause of hospital admission in the elderly. It is characterized by an acute fluctuating impairment of cognitive functions and inattention. Recognition and prompt treatment is crucial to decrease the morbidity and mortality of hospitalized elderly patients. Aim of this study was to evaluate the distribution of common medical conditions related to acute confusional state among elderly hospitalized Patients.

Methods: This was a cross sectional study. We enrolled 380 patients from different medicine wards in Dhaka Medical College Hospital. We included patients with acute confusional state/delirium of less than 7 days duration. The enrolled patients fulfilled the diagnostic criteria of an acute confusional state. A predesigned checklist was used for data collection. Head injury was excluded by history and CT scan of brain. Patients with preexisting illnesses, such as dementia, psychiatric illnesses and recurrent seizures, and any case of poisoning was excluded. All findings were noted and recorded. A written informed consent was taken from the attendant and relatives.

Result: Among 380 patients, most of confusional state developed after the age of 65 years and the mean age was $69+/-7.6$ years. Associated medical conditions were uncontrolled hypertension (63.2\%), fever \& infection (4I.6\%), uncontrolled DM (36.6\%), CVD (28.4\%), CKD \& electrolytes abnormalities (10.5\%), joint diseases \& pain (13.7\%). Most of the patients used plyphormacy (76.6\%) out of which antihypertensive (60\%) were common. The mean duration of presentation was $6.1+1-0.6$ days, and among all patients about $63.7 \%$ were improved, $29.50 \%$ was in persistant symptom of confusion and $6.8 \%$ of them died.
\end{abstract}

Conclusion: Acute confusional state was common after 65 years. Uncontrolled hypertension, fever \& infection, uncontrolled diabetes, CVD, CKD \& electrolytes abnormalities and polypharmacy were found commonly in patients with acute confusional state.

Keywords: Acute confusional state, Elderly, Etiology

DOI: https://doi.org//0.3329/jom.v22il.5/ 390

Copyright: (C) 2021 Rahman.This is an open access article published under the Creative Commons AttributionNonCommercial-NoDerivatives 4.0 International License, which permits use, distribution and reproduction in any medium, provided the original work is properly cited, is not changed in any way and it is not used for commercial purposes.

Received: 10 October, 2020;

Accepted: 22 November, 2020

1. Associate Professor, Department of Medicine, Dhaka Medical College, Dhaka.

2. Medical officer, Rajshahi Medical College and Hospital

3. Medical officer Sir Salimullah Medical College and Mitford Hospital

4. Medical officer, NHS, UK

5. Associate professor, MH Samarita Medical College,Dhaka.

6. Associate professor, Department of Medicine, Sir Salimullah Medical ,College,Dhaka.

7. Indoor Medical Officer, Department of Medicine, Dhaka Medical College Hospital, Dhaka.

8. Junior Consultant, Department of Medicine, Dhaka Medical College Hospital, Dhaka

Corresponding author: Md. Motlabur Rahman, Associate Professor, Department of Medicine, Dhaka Medical College, Dhaka.

\section{Introduction}

Acute confusional state or delirium is a clinical syndrome characterized by disturbed consciousness, cognitive function, or perception. The delirium usually develops over a short period of time (usually hours to days) and it has a tendency to fluctuate during the course of the day. It is often associated with serious adverse outcomes such as death, dementia, and the need for long-term patient care. ${ }^{1,2}$ Incidence of an acute confusional state ranges from $6 \%$ to $56 \%$ in hospitalized patients and nearly $80 \%$ in intensive care unit. ${ }^{3}$ At least $20 \%$ of the 12.5 million patients over 65 years of age hospitalized each year in the US experience complications during hospitalization because of delirium. ${ }^{4}$ It was associated 
with increased functional decline during hospitalization, increased duration of hospital stay, need for long term care, and high short-term mortality rate ${ }^{5}$

Delirium is a common cause of mortality and morbidity in the older population. It adds an extra burden to the care givers and family members. Delirium may be the result of a variety of systemic or cerebral disease or to drug intoxication. The key to the management of cases of an acute confusional state lies in recognizing a cause or other contributing illness and alleviating it. 6

We evaluated the patients of acute confusional state with the objective of identifying the possible causes of acute confusional state along with the evaluation of its prognostic importance.

\section{Methodology}

This cross sectional study was conducted in different medicine wards of Dhaka Medical College Hospital enrolling 380 patients. We collected all the data using a preformed questionnaire.

We included patients with acute confusional state/delirium of less than 7 days duration. The enrolled patients fulfilled the diagnostic criteria of an acute confusional state, according to the confusion assessment method. The confusion assessment method diagnostic algorithm included the following criteria:

(1) An acute onset and fluctuating course

(2) Inattention

(3) Disorganized thinking and

(4) Altered level of consciousness.

The diagnosis of delirium by confusion assessment method (CAM) needed the presence of features 1 and 2 and either 3 or 4.

Head injury was excluded by history and CT scan of brain. Patients with preexisting illnesses, such as dementia, known psychiatric illnesses and recurrent seizures, and any case of poisoning was excluded. All findings were noted and recorded. A written informed consent was taken from the attendant and relatives.

All patients were subjected to a detailed history, neurological and systemic examinations. Information about the drug and alcohol intake was recorded. Cognitive evaluation was performed using the Mini-Mental State Examination (MMSE). All the patients were subjected to routine biochemistry (serum sodium, potassium levels and blood sugar), complete hemogram, liver, renal and thyroid functions, chest X-ray, ultrasound of abdomen, cranial computed tomography and cerebrospinal fluid (CSF) analysis.
All data was analyzed by SPSS version software and Microsoft Excels.

\section{Results}

In this study, 380 elderly patients (age $>60$ years) with acute confusional state were enrolled to find out the etiology. The majority of delirium occurred after the age of 65 years and the mean age was $69( \pm 7.6)$ years, ranging from 60 to 100 years (figure:3)

This study showed male preponderance $(256,67.36 \%)$. Out of 380 patient $256(67.36 \%)$ was male and $124(32.63 \%)$ was female.

Associated medical conditions (Figure 2) were uncontrolled hypertension (63.2\%), fever \& infection (41.6\%), uncontrolled DM (36.6\%), CVD (28.4\%), CKD \& electrolytes abnormalities (10.5\%), joint diseases \& pain (13.7\%). Most of the patients used polypharmacy (76.6\%) out of which multiple antihypertensive $(60 \%)$ were common.

Among all study patients about $63.7 \%$ were improved and completely recovered, $29.50 \%$ was in persistent symptom of confusion, and $6.8 \%$ of them died. (figure: 3 )

Table 1 : Age distribution of the patients $(N=380)$

\begin{tabular}{lcc}
\hline Age groups (years) & Frequency & Percentage \\
\hline $60-70$ & 283 & 74.5 \\
$71-80$ & 59 & 15.5 \\
$81-90$ & 31 & 8.2 \\
$91-100$ and above & 7 & 1.8 \\
\hline
\end{tabular}

Table: 1 shows the distribution of age of the participants. Most of the patients were in age group 60-70 years (283, $74.5 \%)$.

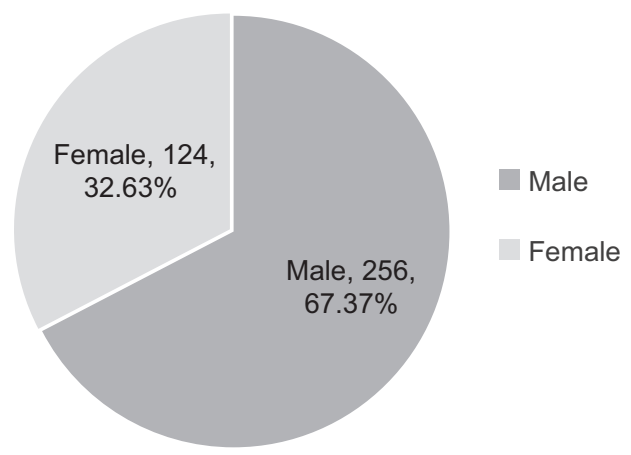

Figure 1: Sex distribution of the patients 
Figure 1 shows sex distribution of the patients. This study showed male preponderance $(256,67.36 \%)$. Out of 380 patient $256(67.36 \%)$ was male and $124(32.63 \%)$ was female.

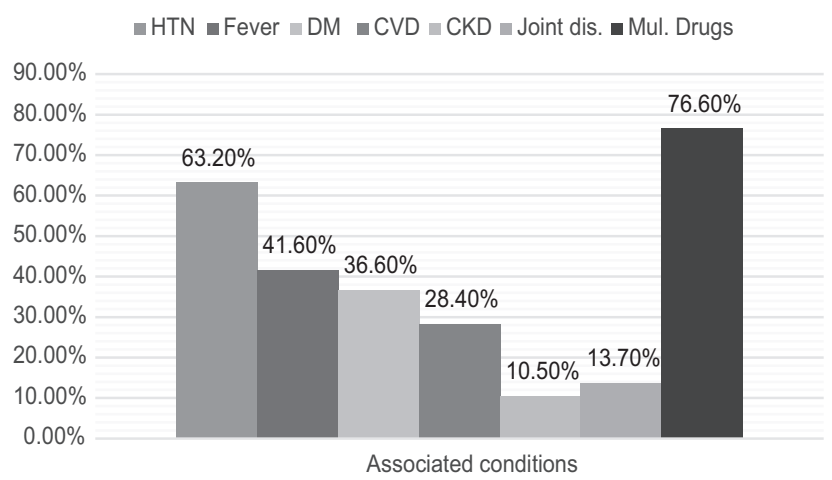

Figure 2: Associated comorbidities ( $\mathrm{N}=380$ shows most of the patients had polypharmacy $(76.60 \%)$ as associated condition and uncontrolled hypertension as the most common associated comorbidity (63.20\%) followed by fever \& infection $(41.60 \%)$.

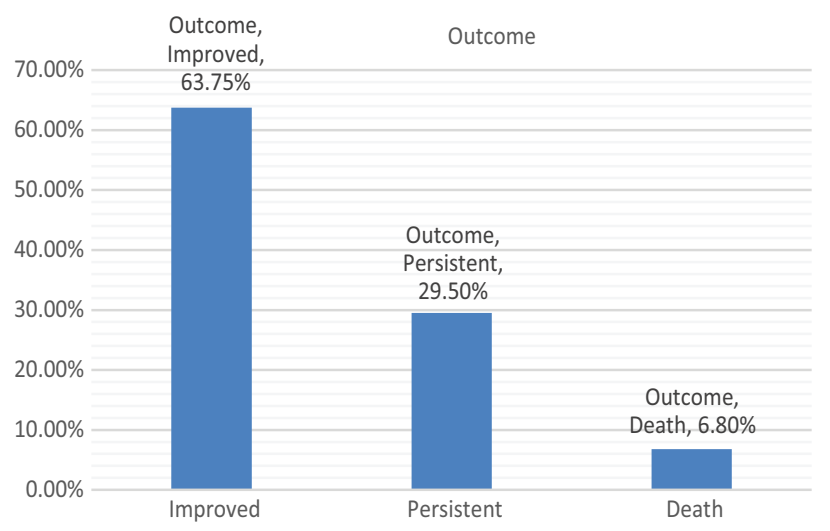

Figure 3: Shows the outcome of the patients $(N=380)$

Figure 3: Shows the outcome of the patients at the end of the study period. Most of the patients got improved (63.75\%), and $29.50 \%$ show persistent symptom of confusion only $6.80 \%$ of patients expired.

\section{Discussion}

We observed that identifying the precipitating factor is important because treatment of precipitating factors influenced the overall outcome in patients with acute confusional state. Both number of deaths and duration of hospital stay were longer if a precipitating factor was not identified. Occurrence of delirium, in fact, reflects an underlying brain dysfunction, which almost invariably results following a systemic or brain disorder or to intoxication from medication. Delirium often has an adverse prognostic impact on functional and cognitive outcome, as well as on morbidity and mortality. ${ }^{7,8}$

In this study, 380 elderly patients (age $>60$ years) with acute confusional state were enrolled to find out the etiology. The majority of delirium occurred after the age of 65 years and the mean age was $69( \pm 7.6)$ years, ranging from 60 to 100 years (figure:3) (Table 1) which was consistent with the study done by Sumji S et al, where majority of the patients were in age group of $61-70$ years and mean age $60 \pm 17.80$ years. ${ }^{9}$ Another study was conducted by Kaur $J$ et al, they found that Most patients were in the age group of 61-80 years, mean age was $54.77 \pm 18.50$ years $^{10}$

In our study there was male predominance. Out of 380 patient $256(67.36 \%)$ was male and $124(32.63 \%)$ was female. Similarly, in the study done by Rai D et al,where they found $61.5 \%$ were males and $38 \%$ were females. ${ }^{11}$

In this study most common medical condition contributes to delirium were uncontrolled hypertension $(63.2 \%)$, then fever $\&$ infection (41.6\%), uncontrolled DM (36.6\%), CVD (28.4\%), CKD \& electrolytes abnormalities (10.5\%), joint diseases \& pain (13.7\%). (Figure 2)

Similar finding was observed by a study by Kaur J et al ,they found ACS was associated with one or the other predisposing factors in $85 \%$ of the patients, only $15 \%$ of the patients had no such risk factors. Out of total 100 patients, $19 \%$ of the patients were hypertensive, $17 \%$ had uncomplicated diabetes, CKD in $13 \%$ of patients, CLD in $13 \%$ patients, complicated diabetes in $6 \%$ of the patients, multidrug abuse in $6 \%$, malignancy $6 \%$, alcohol usage in $4 \% .^{10}$

Another study was conducted by Rai $D$ et al, they also mention that hypertension was the most common $(n=14$, $26.92 \%$ ) associated condition. Four patients, with cerebrovascular accidents, had multiple infarcts. In that study, $33(66.5 \%)$ patients had a possible precipitating factor and in remaining patients a precipitating cause of acute confusional state was notestablished ${ }^{11}$

In the study conducted by Sumji S et al, the most common final diagnosis was CVA 161 patients (25.6\%) followed by metabolic disturbances group 157 (24.9\%), then Sepsis/ infections $117(18.5 \%)$, seizures 83 (13.0\%), intracranial infections (meningitis, encephalitis, meningoencephalitis, and brain abscess) 32 patients (5.0\%), then cardiovascular/ shock group $31(4.9 \%)$, ICSOL $16(2.6 \%)$, poisoning 8 (1.3\%), SDH 9 (1.5\%), and unknown/miscellaneous 17 $(2.7 \%) .^{12}$ 
$V B$ Kalish et al have reported a wide array of conditions, associated with delirium, including, infection, ulcer, sever illness, etc. many of which may present with fever. ${ }^{13}$

Kim et al. reviewed the medical records of 260 delirium patients and 77 control patients. They investigated age, sex, and risk factors for delirium in the total delirium group $(n=260)$, the delirium medical subgroup $(n=142)$, and the delirium surgical subgroup $(n=118)$. Logistic regression analysis adjusting for age and sex showed that in the delirium group risk factors were lower plasma albumin, uncontrolled hypertension, mechanical ventilation, and antipsychotic drug use. Plasma sodium level and uncontrolled hypertension were important risk factors for the delirium medical subgroup. Stroke history, uncontrolled hypertension, ICU care, and medication were important risk factors for the delirium surgical subgroup. ${ }^{14}$

In our observation we found that most of the patients used polypharmacy $(76.6 \%)$ out of which multiple antihypertensive medications (60\%) were common. Hein et al found that, nearly $25 \%$ of hospitalized patients had delirium. The Confusion Assessment Method was positive in $69 \%$ of patients receiving polypharmacy and in $30 \%$ of those not receiving polypharmacy, a relative risk of $2.33 .{ }^{15}$

The mean duration of presentation of our study patient and hospital staying time was $6.1+/-0.6$ days. The study by $R a i$ $D$ et al The mean duration of hospital stay was $10.73 \pm 3.6$ days (range 5-21 days). 18 (34.6\%) of our patients died, of these in 10 patients we could not find a precipitating cause.

Among all our study patient about $63.7 \%$ were improved and completely recovered, $29.50 \%$ was with persistent symptom of confusion and $6.8 \%$ of them died. Comparable to the study done by Grover $S$ et al where $65.9 \%$ patients had improved, $8.8 \%$ recovered, $6.6 \%$ died. ${ }^{16}$

Several studies have suggested that delirium is associated with risk of dementia and also acceleration of decline in existing dementia. Davis et al recently noted that, delirium was associated with general cognitive decline, with an 8fold increase in incident dementia and accelerated decline in MMSE scores. ${ }^{17}$ Early recognition and prompt treatment are essential to prevent future cognitive decline in these patients.

Nonetheless, we had some limitations in this study. This was only a hospital based observational study and might not have reflected the real scenario within the community. Moreover, we could not measure the odd ratio for individual risk factors due to nature of the study. This warrants further case control studies to confirm the level of association.

\section{Conclusion}

Acute confusional state was common after 65 years. Uncontrolled hypertension, fever \& infection, uncontrolled diabetes, CVD, CKD \& electrolytes abnormalities and polypharmacy for different diseases were present in many patients with acute confusional state. Targeted interventions could substantially reduce the overall burden of delirium as well as it could reduce the hospital stay and mortality of hospitalizd elderly patients.

\section{References}

1. Young J, Murthy L, Westby M, Akunne A, O'Mahony R. Guideline Development Group. Diagnosis, prevention, and management of delirium: Summary of NICE guidance. BMJ 2010; 341:c3704.

2. McCusker J, Cole M, Dendukuri N, Han L, Belzile E. The course of delirium in older medical inpatients: A prospective study. J Gen Intern Med. 2003;18:696-704.

3. Fong TG, Tulebaev SR, Inouye SK. Delirium in elderly adults:Diagnosis, prevention and treatment. Nat Rev Neurol. 2009; 5:210-20.

4. Fong TG, Tulebaev SR, Inouye SK. Delirium in elderly adults: diagnosis, prevention and treatment. Nat Rev Neurol. 2009 Apr; 5(4):210-20.

5. Hénon H, Lebert F, Durieu I, Godefroy O, Lucas C, Pasquier F, et al. Confusional State in Stroke. Stroke. 1999 Apr 1; 30(4):773-9.

6. Burns A, Gallagley A, Byrne J. Delirium. J Neurol Neurosurg Psychiatry. 2004;75:362-7.

7. Leentjens AF, van der Mast RC. Delirium in elderly people: An update. Curr Opin Psychiatry. 2005;18:325-30. 8. Han JH, Shintani A, Eden S, Morandi A, Solberg LM, Schnelle $\mathrm{J}$, et al. Delirium in the emergency department: An independent predictor of death within 6 months. Ann Emerg Med. 2010;56:244-2521.

9. Sumji S, Wani MI, Mohi-ud-Din M. Prevalence and Etiological Profile of Patients with Acute Confusional State. Int J Sci Stud. 2018;6(5):24-9.

10. Jaspreet K, Gurinder M, Nayyar S. B, Ranjeet K. A study of etiological profile of acute confusional state. Int JAdv Med. 2020 Jan;7(1):138-144

11. Rai D, Garg RK, Malhotra HS, Verma R, Jain A, Tiwari SC, et al. Acute confusional state/delirium: An etiological and prognostic evaluation. Ann Indian Acad Neurol. 2014 Jan; 17(1):30-4.

12. Sajad Sumji, Mohd Iqbal Wani1, Muzzafar Mohi-ud-Din. Acute confusional state/delirium: An etiological and prognostic evaluation, Annals of Indian Academy of Neurology. January-March 2014, Vol 17, Issue 1 
13. Kalish VB, Gillham JE, Unwin BK. Delirium in Older Persons: Evaluation and Management. AFP. 2014 Aug $1 ; 90(3): 150-8$.

14. Kim H, Chung S, Joo YH, Lee JS. The major risk factors for delirium in a clinical setting. Neuropsychiatr Dis Treat. 2016 Jul 21;12:1787-93.

15. Hein C, Forgues A, Piau A, Sommet A, Vellas B, Nourhashémi F. Impact of Polypharmacy on Occurrence of Delirium in Elderly Emergency Patients. Journal of the
American Medical Directors Association. 2014 Nov 1;15(11):850.e11-850.e15.

16. Grover S, Malhotra S, Bharadwaj R, Bn S, Kumar S. Delirium in children and adolescents. Int J Psychiat Med. 2009 Jun;39(2):179-87.

17. Davis DH, Muniz Terrera G, Keage H, Rahkonen T, Oinas $\mathrm{M}$, Matthews FE, et al. Delirium is a strong risk factor for dementia in the oldest-old: A population-based cohort study. Brain 2012;135:2809-16. 